\title{
Iterative H.264 Source and Channel Decoding Using Sphere Packing Modulation Aided Layered Steered Space-Time Codes
}

\author{
Nasruminallah, M. El-Hajjar and L. Hanzo \\ School of ECS, University of Southampton, SO17 1BJ, UK. \\ http: //www-mobile.ecs.soton.ac.uk, \\ Email: $\{1 \mathrm{~h}\} @ e c s . s o t o n . a c . u k$
}

\begin{abstract}
The conventional two-stage turbo-detection schemes generally suffer from a Bit Error Rate (BER) floor. In this paper we circumvent this deficiency by proposing a three-stage turbo detected Sphere Packing (SP) modulation aided Layered Steered Space-Time Coding (LSSTC) scheme for H.264 coded video transmission over correlated Rayleigh fading channels. The soft-bit assisted H.264 coded bit-stream is protected using lowcomplexity short-block codes (SBCs), combined with a rate-1 recursive inner precoder is employed as an intermediate code which has an infinite impulse response and hence beneficially spreads the extrinsic information across the constituent decoders. This allows us to avoid having a BER floor. Additionally, the convergence behaviour of this serially concatenated scheme is investigated with the aid of Extrinsic Information Transfer (EXIT) Charts. The proposed system exhibits an $E_{b} / N_{0}$ gain of about $12 d B$ in comparison to the benchmark scheme carrying out iterative source-channel decoding as well as Layered Steered Space-Time Coding (LSSTC) aided Sphere Packing (SP)demodulation, but dispensing with the optimised SBCs.
\end{abstract}

\section{Motivation AND BACKGROUND}

Shannon's source-channel separation principle [1] states that under idealised, but only asymptotically valid conditions source coding and channel coding schemes may be designed independently. However, in reality the source codes designed without taking into account the presence of channel decoding errors tend to have a poor performance, owing to the finite length, finite complexity source and channel codes employed. In a high compression source codec a low number of erroneous bits in an entropy coded sequence typically is mapped to a high video distortion in the reconstructed sequence. This results in low target error rate requirements for channel coding, in order to prevent the source coded video from becoming unduly impaired. In such a situation, joint source-channel decoding (JSCD) results in substantial performance improvements at a given channel coding rate, by exploiting any residual redundancy inherent in the resultant bit-stream after source coding. In recent years, the turbo-principle was extended to JSCD by invoking iterative source-channel decoding (ISCD) by iteratively exchanging extrinsic information between two or more concatenated codes. In [2,3], it was revealed that ISCD schemes indeed exhibit superior bit-error correction capability incomparison to those of the non-iterative schemes. However, the number of useful iterations is typically limited to two or three iterations $[4,5]$ due to the limited residual redundancy left in the source coded stream. In [4], it was shown that the achievable ISCD performance can be improved by introducing artificial redundancy in the source coded stream. Therefore, in our proposed scheme we advocate a novel class of short block codes [6] invoked, in order to improve the attainable iterative decoding performance. A turbo-detected multidimensional Sphere Packing (SP) modulation scheme using two transmit antennas combined with Differentially encoded Space-Time Spreading (DSTS) is presented in [7]. DSTS is a non-coherent
MIMO scheme capable of providing a diversity gain, while attaining no multiplexing gain for the case of two transmit antennas and a throughput loss in the four transmit antennas case compared to a single transmit antenna system. However, throughput improvements can be achieved using a multi-layer MIMO structure designed for achieving a multiplexing gain, which is known as the vertical bell labs layered space-time (V-BLAST) scheme [8]. More explicitly, the advantage of VBLAST is that it is capable of providing an increased effective bit-rate without any increase in the transmitted power or in the system's bandwidth. By contrast, a high transmit diversity gain can be achieved using Alamouti's low-complexity spacetime code (STC) [9]. On the other hand, beamforming [10] provides an effective technique of reducing the multiple-access interference (MAI), where the antenna gain is increased in the direction of the target user, while reducing the gain towards the interfering users. In order to combine the benefits of the above-mentioned three MIMO schemes, in our proposed system design, we considered an amalgamated transmitter design, jointly exploiting the merits of V-BLAST, STC and beamforming, in order to achieve a high diversity gain, a high throughput as well as a beamforming gain which we referred to as a layered steered space-time code (LSSTC).

The rest of the paper is organised as follows. An overview of our system model is provided in Section II. Section III portrays our employed video source codecs followed by the details of the soft-bit source decoding (SBSD) procedure employed. The performance of the proposed system is characterised with the aid of EXIT chart analysis in Section IV, while the overall performance results are presented in Section V. Finally, we offer our conclusions in Section VI.

\section{System OVERVIEW}

\section{A. Transmitter Model}

The schematic of the proposed scheme is illustrated in Figure 1. The architecture seen in Figure 1 has $N_{t}=4$ transmit Antenna Arrays (AA), which are spaced sufficiently far apart in order to encounter independent fading and hence to achieve transmit diversity. Each AA is equipped with $L$ number of elements that are spaced at a distance of $d=\lambda / 2$ in order to achieve beamforming gain, where $\lambda$ represents the carrier's wavelength. Furthermore, the receiver is equipped with $N_{r}=$ 4 receiver antennas. According to Figure 1, the video stream is encoded using the H.264 video codec and the resultant bit-stream $x_{k}$ is encoded into the bit-string $s$ using a rate$\frac{1}{3}$ SBC scheme, where the $i^{t h}$ video frame is partitioned into $N$ source coded symbols and each symbol $y_{n, k}$ consists of $M$ source coded bits $y_{n, k}(m), m=1, \ldots M$. More specifically, in our case $M=2$-bit input symbols are encoded by the rate- $\frac{1}{3}$ SBCs, resulting in $M^{\prime}=6$-bit SBC coded symbols. The SBC encoded bits $s$ are interleaved using a random 


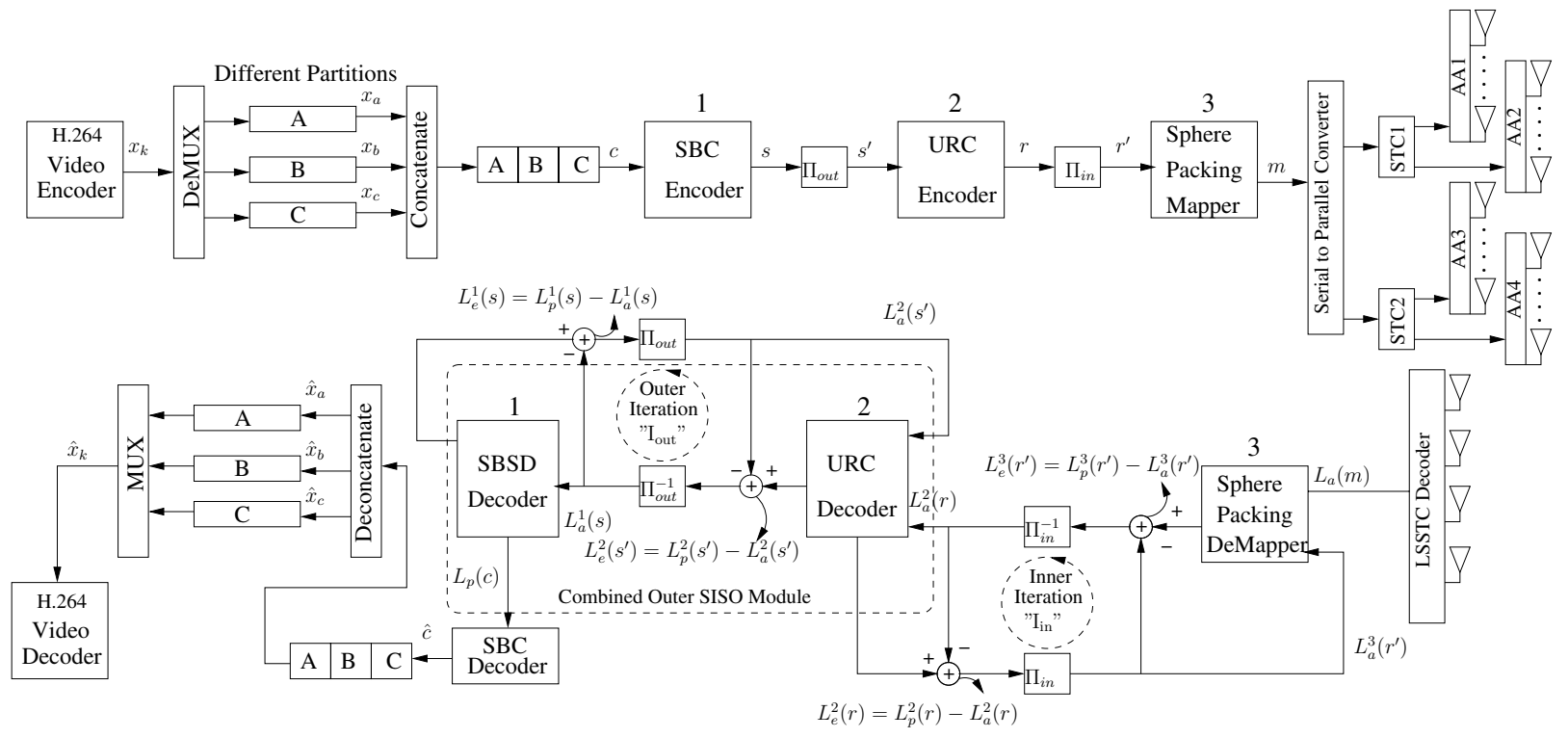

Fig. 1. The Proposed three-stage Iterative Source-Channel Decoding System Model.

bit interleaver $\Pi_{\text {out }}$, which are then further encoded by the Unity Rate Coder (URC) [11]. Following URC encoding, the resultant bits $r$ are interleaved by the second random bit interleaver $\Pi_{i n}$ into $r^{\prime}$ and are passed to the Sphere Packing (SP) modulator $[11]^{1}$. The diversity product of the LSSTC scheme is determined by the minimum Euclidean distance of all legitimate transmitted vectors. Therefore, the SP modulator provides the benefit of jointly considering the space-time symbols of the LSSTC scheme, so that they are represented by a single phaser point selected from the SP constellation having the best known minimum Euclidean distance in the real-valued space. Since we invoke a twin-AA aided STC scheme, the $\mathrm{SP}$ design required is 4-dimensional. Assuming that there are $L_{s p}$ legitimate vectors, the transmitter then has to choose the modulated signal from these $L_{s p}$ legitimate symbols to be transmitted over the two AAs. More specifically, the SP modulator maps $B$ number of coded bits $b=b_{0}, \ldots b_{B-1} \in 0,1$ to a SP symbol $v \in V$ so that we have $v=\operatorname{map}_{s p}(b)$, where $B=\log _{2}\left(L_{s p}\right)$, and $L_{s p}$ represents the set of legitimate SP constellation points, as detailed in [11]. In this contribution, we considered $B=\log _{2}(16)=4$ channel coded bits per SP symbol. Additionally, we consider transmission over a temporally correlated narrowband Rayleigh fading channel, associated with a normalised Doppler frequency of $f_{D}=$ $f_{d} T_{s}=0.01$, with $f_{d}$ being the Doppler frequency and $T_{s}$ the symbol duration. The transmitted signal is contaminated by the complex-valued Additive White Gaussian Noise (AWGN) of $n=n_{I}+j n_{Q}$, where $n_{I}$ and $n_{Q}$ are two independent zeromean Gaussian random variables having a variance of $N_{0} / 2$ per dimension, with $N_{0} / 2$ represents the double-side noise power spectral density expressed in $W / H_{z}$.

B. Receiver Model

At the receiver, the extrinsic information represented in the form of LLRs is iteratively exchanged between the SP demapper, the A Posteriori Probability Soft-In Soft-Out (APP SISO) decoder of the URC-decoder and the SBC decoder, in order to assist each other in approaching the point of perfect

\footnotetext{
${ }^{1}$ The role of the SP scheme is to combine the conventional QPSK symbols of Alamouti's multiple antenna based schemes into a joint SP symbol. The benefit of this is that this way we can jointly design the mutiple antennas' space-time symbols, which allows us to improve the attainable diversity gain at the cost of an increased decoder complexity.
}

convergence at the $(1,1)$-point of the EXIT-chart as detailed in [11]. The respective bit-LLRs of the constituent codes are represented by $L($.$) in the proposed three-stage system design$ of Figure 1, and are differentiated by the superscripts of 1,2 and 3, indicating the outer SBC decoder, the intermediate URC decoder and the inner SP decoder, respectively. Additionally, the specific type of the LLRs-i.e. a priori, a posteriori and extrinsic information is indicated by the subscript $a, p$ and $e$, respectively.

The inner iteration consists of extrinsic information exchange between the outer SP demapper and the intermediate URC decoder of Figure 1. The received complex valued symbols corresponding to $B$ number of URC-coded bits per SP symbol are demapped to their LLR representations [12]. The extrinsic LLR values $L_{e}^{3}\left(r^{\prime}\right)$ are generated by subtracting the a priori information $L_{a}^{3}\left(r^{\prime}\right)$ provided by the URC decoder from the a posteriori LLR value $L_{p}^{3}\left(r^{\prime}\right)$ at the output of the SP-demapper, as shown in Figure 1. The LLRs $L_{e}^{3}\left(r^{\prime}\right)$ are deinterleaved by the softbit interleaver $\Pi_{i n}$ of Figure 1 and are passed to the URC-decoder in order to produce the a posteriori LLR values $L_{p}^{2}(r)$ using the MAP algorithm [13] for the URC-encoded bits. The extrinsic LLR values $L_{e}^{2}(r)$ of the URC-encoded bits $r$ are obtained by subtracting the a priori LLRs $L_{a}^{2}(r)$ input to the URC-encoder from the URC generated a posteriori LLRs $L_{p}^{2}(r)$. Following interleaving, the resultant extrinsic LLRs $L_{e}^{2}(r)$ are fed back to the SPdemapper as the a priori information $L_{a}^{3}\left(r^{\prime}\right)$. This a priori information is exploited by the SP demapper for the sake of providing improved extrinsic information for the URC decoder in the successive iterations.

The outer iterations are comprised of exchanging extrinsic information between the SBSD and URC decoder of Figure 1. First, the extrinsic LLRs $L_{e}^{2}\left(s^{\prime}\right)$ produced by subtracting the a priori information $L_{a}^{2}\left(s^{\prime}\right)$ from the a posteriori LLRs $L_{p}^{2}\left(s^{\prime}\right)$ generated by the URC decoder are deinterleaved using the soft-bit interleaver $\Pi_{\text {out }}$ of Figure 1 and passed to the SBSD. The SBSD computes the a posteriori LLRs $L_{p}^{1}(s)$ and subtracts the input a priori LLRs $L_{a}^{1}(s)$ from it in order to produce the extrinsic LLRs $L_{e}^{1}(s)$. These extrinsic LLRs are interleaved and fed back as a priori information to the URC decoder for the sake of generating improved extrinsic LLRs 
in the subsequent iterations. In the system design of Figure 1 we define a system iteration $I_{\text {system }}$ as that composed of one inner iteration followed by two outer iterations.

\section{SOURCE CODING AND EXTRINSIC INFORMATION GENERATION PROCEDURE}

The "Akiyo" video sequence [14] consisting of $45(176 \times$ 144)-pixels Quarter Common Intermediate Format (QCIF) frames and encoded using the H.264/AVC JM 13.2 reference video codec at 15 frames-per-second (fps) at the target bitrate of $64 \mathrm{kbps}$ was used as our test sequence. Each QCIF frame was partitioned into 9 slices and each slice was composed of $11 \mathrm{MBs}$, of a row within a QCIF frame. The resultant video encoded clip consists of an intra-coded 'I' frame followed by 44 predicted ' $P$ ' frames, implying that an 'I' frame was inserted in the video sequence after every 45 frames corresponding to 3 seconds at 15 frames/sec, in order to curtail error propagation. Additional, source codec parameters include the employment of quarter-pixel motion estimation, intra-frame MB update and the use of Universal Variable Length Coding (UVLC) type entropy coding. Furthermore, error resilience features such as Data Partitioning (DP) and intra-frame coded MB update of three randomly distributed MBs per QCIF frame were also incorporated. The insertion of bi-directionally predicted ' $\mathrm{B}$ ' pictures results in an unacceptable loss of lip-synchronisation owing to its delay and hence was avoided. Moreover, instead of multiple frame based motion trajectory prediction, the motion search was restricted to the immediately preceding QCIF frame in order to reduce the computational complexity of the video decoder. Similarly, the employment of Flexible Macroblock Ordering (FMO) was turned off, because despite its substantial increase in computational complexity it typically resulted in modest video performance improvements in lowmotion video-telephony, when using for example the "Akiyo" video test sequence. The remaining system parameters of our experimental setup are listed in Table II.

In order to attain a beneficial iterative decoding performance gain, we utilised the appropriately modified soft-bit source decoder (SBSD) philosophy of $[15,16]$, which exploits the residual redundancy in the speech coded bit-stream, as a priori information for computing the extrinsic Logarithmic Likelihood Ratios (LLRs) [17]. The SBSD of the source encoder partitions the video bitstream into short time frames, which are labeled with the video frame index $i$. From each of the video frames a parameter set $Y_{i}$ consisting of $N$ scalar source codec parameter patterns $y_{i, n}, n=1,2 \cdots, N$ is generated. The extrinsic information generated by the SBSD is the information gleaned for each data bit $y_{i, n}(m), m=$ $1,2 \cdots, M$, from all other data bits of the parameter pattern. In a scenario where all bits of the bit pattern $y_{i, n}(m)$ except for $y_{i, n}(\lambda)$ is perfectly known at the receiver, the extrinsic LLR can be expressed as:

$$
L_{e}^{1}\left(y_{i, n}(\lambda)\right)=\log \frac{\sum_{m=1, m \neq \lambda}^{M} P\left[y_{i, n}(m) \mid y_{i, n}(\lambda)=+1\right]}{\sum_{m=1, m \neq \lambda}^{M} P\left[y_{i, n}(m) \mid y_{i, n}(\lambda)=-1\right]} .
$$

Normally, the bits providing the extrinsic LLR-values of the bit considered are not known perfectly. In such a situation the reliabilities of all the possible realizations of the contributing bits has to be determined based on the a priori LLR-values
$L_{a}^{1}\left[y_{i, n}(\lambda)\right]$ provided at the input of the SBSD, as shown in Figure 1, which is given as:

$$
R\left[y_{i, n}(\lambda)\right]=\exp \sum_{m=1, m \neq \lambda}^{M} \frac{y_{i, n}(\lambda)}{2} \cdot L_{a}^{1}\left[y_{i, n}(\lambda)\right] .
$$

Observe in (1) that the bit under consideration is excluded from the present bit pattern. After calculating (2) for all possible realization of the $(M-1)$ contributing bits, Equation (1) can be extended to:

$$
\begin{gathered}
L_{e}^{1}\left(y_{i, n}(\lambda)\right)= \\
\left.\log \frac{\sum_{m=1, m \neq \lambda}^{M} P\left[y_{i, n}(m) \mid y_{i, n}(\lambda)=+1\right] \cdot R\left[y_{i, n}(\lambda)\right]}{\sum_{m=1, m \neq \lambda}^{M} P\left[y_{i, n}(m) \mid y_{i, n}(\lambda)\right.}=-1\right] \cdot R\left[y_{i, n}(\lambda)\right]
\end{gathered}
$$

In this paper we considered two different schemes, which differ in the choice of the outer SBC code employed. Specifically, we considered EXIT-chart optimised SBCs having a minimum Hamming distance of $d_{H, \text { min }}=3$ and an equivalent-rate nonoptimised SBC based benchmarker having $d_{H, \text { min }}=1$, as given in Table I. We refer to these two schemes as the LSSTCSP-URC-SBC and LSSTC-SP-URC-SBC ${ }^{\star}$ arrangements.

$$
\text { TABLE I }
$$

SBCS WITH CORRESPONDING SYMBOLS AND $d_{H, \text { min }}$

\begin{tabular}{|l|l|c|}
\hline SBC Type & Symbols in Decimal & $d_{H, \text { min }}$ \\
\hline \hline Un-optimised Rate- $\frac{1}{3} \mathrm{SBC}_{[2,6]}^{\star}$ & $\{0,16,32,48\}$ & 1 \\
\hline Optimised Rate- $\frac{1}{3} \mathrm{SBC}_{[2,6]}$ & $\{0,22,41,63\}$ & 3 \\
\hline \hline
\end{tabular}

TABLE II

SYSTEMS PARAMETERS

\begin{tabular}{|l|l|l|l|}
\hline System Parameters & Value & System Parameters & Value \\
\hline \hline Source Coding & H.264/AVC & No of MB's/Slice & 11 \\
Bit Rate (Kbps) & 64 & Intra-frame MB & 3 \\
Frame Rate (fps) & 15 & update/frame & \\
No of Slices/frame & 9 & Over-all Code Rate & $1 / 3$ \\
\hline Intermediate code & URC & Channel & $\begin{array}{l}\text { Correlated } \\
\text { Rayleigh }\end{array}$ \\
\hline $\begin{array}{l}\text { Modulation Scheme } \\
\text { Number of }\end{array}$ & SP & & Fading \\
Transmitter AA, $N_{t}$ & 4 & Normalised & \\
$\begin{array}{l}\text { Number of } \\
\text { Receiver Antennas, } N_{r} \\
\text { Interleaver Length }\end{array}$ & 4 & $\begin{array}{l}\text { Doppler } \\
\text { Frequency }\end{array}$ & 0.01 \\
\hline
\end{tabular}

\section{ITERATIVE DETECTION AND EXIT CHART ANALYSIS}

The main objective of employing EXIT charts [18] is to predict the convergence behaviour of the iterative decoder by examining the evolution of the input/output mutual information (MI) exchange between the constituent decoders of Figure 1. In our proposed three stage system setup, the intermediate URC decoder of Figure 1 receives its input from and provides output to both the SP-mapper and the SBSD. Generally, in EXIT chart analysis, for the symbol $s$ having the a priori LLR value $L_{a}(s)$, the mutual information (MI) is denoted by $I_{., A}(s)$, while the MI between the extrinsic LLR $L_{\dot{e}}(s)$ and the corresponding symbol $s$ is denoted by $I_{., E}(s)$. The MI associated with the corresponding symbols of one of the three constituent decoders is differentiated by replacing the subscript (.) with the corresponding subscript 1,2 and 3 for the SBC, URC and SP-mapper, respectively. Therefore, 
the URC decoder is provided with two a priori inputs. The first a priori input $L_{a}^{2}(r)$ corresponding to the coded bits $r$ is provided by the SP demapper. The second a priori input $L_{a}^{2}\left(s^{\prime}\right)$ corresponding to the data bits $s^{\prime}$ is provided and fed back by the SBSD of Figure 1 .

Similarly, the URC decoder generates two extrinsic outputs corresponding to the data bits $s^{\prime}$ and $r$, which are represented as $L_{e}^{2}\left(s^{\prime}\right)$ and $L_{e}^{2}(r)$, respectively. Furthermore, the EXIT characteristics of the URC decoder can be described by the EXIT functions $F_{s}^{\prime}\left[I_{2, A}\left(s^{\prime}\right), I_{2, A}(r)\right]$ and $F_{r}\left[I_{2, A}\left(s^{\prime}\right), I_{2, A}(r)\right]$ of the URC decoder. By contrast, the SBSD and the SP demapper only receives input from and provides output for the URC decoder. Therefore, their corresponding EXIT functions are $F_{s}\left[I_{1, A}(s)\right]$ and $F_{r^{\prime}}\left[I_{3, A}\left(r^{\prime}\right), E_{b} / N_{0}\right]$, respectively.

For the EXIT chart analysis of system of Figure 1 we considered the intermediate URC decoder and the SBSD as a single combined outer Soft Input Soft Output (SISO) module. The EXIT chart of the proposed benchmarker LSSTC-SP$\mathrm{URC}^{-S B C}{ }^{\star}$ scheme along with the EXIT curves of the SP demapper recorded for the $E_{b} / N_{0}$ values of 1 to $6 \mathrm{~dB}$ is shown in Figure 2. As observed in Figure 2, the EXIT cure of the combined outer SISO module constituted by the LSSTC-SPURC-SBC $^{\star}$ scheme cannot reach the $(1,1)$ point of perfect convergence in the EXIT chart. Since this system is unable to provide an open EXIT tunnel, an infinitesimally low BER cannot be achieved.

By contrast, the outer EXIT curve of the combined SISO module recorded for the LSSTC-SP-URC-SBC scheme is shown in Figure 3 along with the EXIT curves of the SP demapper recorded for various $E_{b} / N_{0}$ values. Figure 3 shows that the joint EXIT curve of the SBSD and URC decoder in the LSSTC-SP-URC-SBC arrangement succeeds in reaching the $(1,1)$ point of the EXIT chart. Figures 2 and 3 also provide the Monte-Carlo-simulation based decoding trajectories of the proposed system at the $E_{b} / N_{0}$ values of 1 to $6 \mathrm{~dB}$. These trajectories were recorded by acquiring the mutual information at the input and output of both the inner SP demapper and of the joint outer SISO module during the bit-by-bit MonteCarlo simulation of the iterative decoding algorithm. Observe from the decoding trajectories of Figure 3 that for an $E_{b} / N_{0}$ value of $1 d B$ the LSSTC-SP-URC-SBC scheme becomes capable of achieving the highest possible extrinsic information of $I_{E}$ (outer) $=1$ during the iterative decoding process. However, the LSSTC-SP-URC-SBC ${ }^{\star}$ is unable to achieve this goal due to the intersection of the inner and outer decoders' EXIT curves.

\section{System Performance Results}

The attainable performance of our proposed system employing $N_{t}=4$ transmit $\mathrm{AAs}$ and $N_{r}=4$ receive antennas ischaracterisedd in this section. We considered the SP modulation scheme of [11] associated with $L=16$ sphere-packing modulated symbols, while employing AntiGray Mapping (AGM) ${ }^{3}$ for the source bits-to-SP symbol mapping. We considered various numbers of system iterations $I_{\text {system }}$, in order to evaluate its effect on the performance of the system. Additionally, for the sake of increasing the confidence in our results, we repeated each 45 -frame video transmission experiment 160 times with a specific number of system iterations and averaged the generated results.

\footnotetext{
${ }^{3}$ Any bit-to-symbol mapping scheme different from the Gray-mapping is referred to as an anti-Gray mapping (AGM).
}

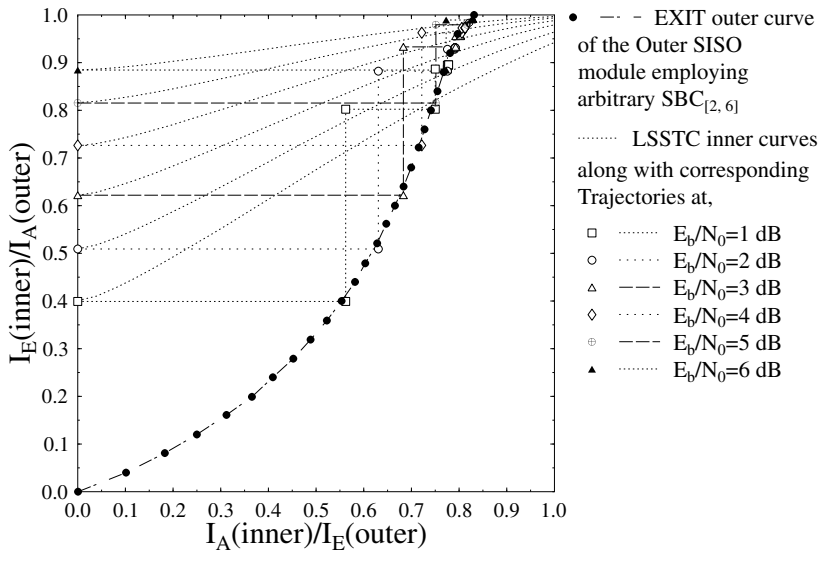

Fig. 2. The EXIT chart and simulated decoding trajectories of the LSSTCSP-SBC ${ }^{\star}$ scheme at $E_{b} / N_{0}=1$ to $6 \mathrm{~dB}$.

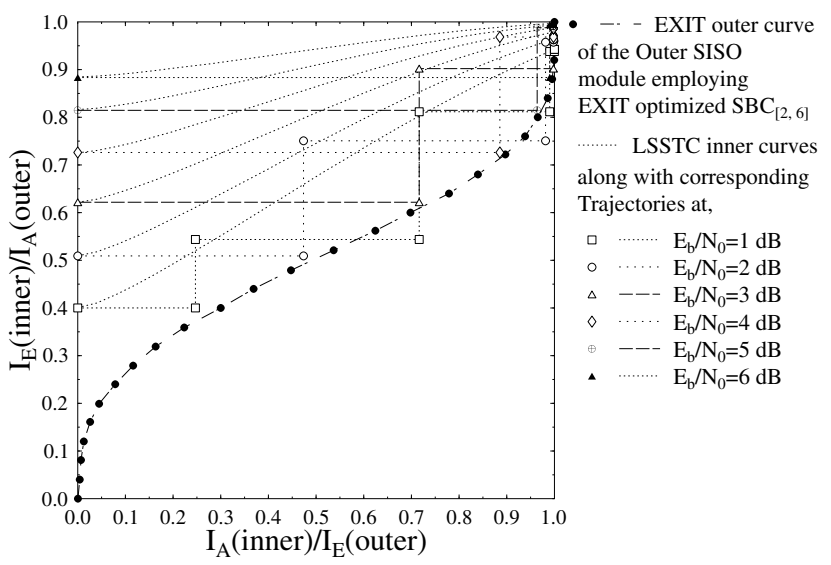

Fig. 3. The EXIT chart and simulated decoding trajectories of the LSSTC$\mathrm{SP}-\mathrm{SBC}$ scheme at $E_{b} / N_{0}=1$ to $6 \mathrm{~dB}$.

The BER performance of the error protection schemes employed is presented in Figure 4. Its evident from Figure 4, that the LSSTC-SP-URC-SBC scheme using $I_{\text {system }}=5$ system iterations results in the best BER performance, when compared to $I_{\text {system }}=4$ and 3 for the same error protection scheme. Additionally, it can be seen that the LSSTC-SP-URC$\mathrm{SBC}^{\star}$ scheme results in the worst BER performance due to its inability to provide perfect iterative decoding convergence.

Additionally, the $P S N R$ versus $E_{b} / N_{0}$ curve of the proposed error protection schemes is portrayed in Figure 5. It may be observed in Figure 5 that the LSSTC-SP-URC-SBC scheme employing rate- $\frac{1}{3}$ SBCs having $d_{H, \min }=3$ and $I_{\text {system }}=5$ system iterations results in the best PSNR performance across the entire $E_{b} / N_{0}$ region considered. It is also observed in Figure 5 that the employment of non-optimised rate- $\frac{1}{3} \mathrm{SBC}$ results in the worst $P S N R$ performance, when using iterative decoding at the same overall code rate of $1 / 3$, as seen in Table III. Quantitatively, when using the LSSTC-SP-URCSBC scheme of Table III, an $E_{b} / N_{0}$ gain of upto $12 d B$ may be achieved relative to the LSSTC-SP-URC-SBC ${ }^{\star}$ scheme at the PSNR degradation point of $1 d B$, as shown in Figure 5.

Finally, the subjective video quality of the error protection schemes employed is characterised in Figure 6. In order to have a pertinent subjective video quality comparison, the video frames presented in Figure 6 were obtained by repeated retransmission of the received video sequence using $I_{\text {system }}=$ 
5 system iterations 30 times. Observe from Figure 6 that an unimpaired video quality is attained by the LSSTC-SP-URCSBC scheme at an $E_{b} / N_{0}$ value of $2 d B$. However, video impairments persist for the LSSTC-SP-URC-SBC ${ }^{\star}$ scheme even at the relatively high $E_{b} / N_{0}$ values of $11 d B, 11.5 d B, 12 d B$ and $12.5 d B$, as shown in Figure 6.

TABLE III

CODE RATES FOR DIFFERENT ERROR PROTECTION SCHEMES

\begin{tabular}{|l|c|c|c|}
\hline \multirow{2}{*}{ Error Protection Scheme } & \multicolumn{3}{|c|}{ Code Rate } \\
\cline { 2 - 4 } & SBC type & Intermediate code & Overall \\
\hline \hline LSSTC-SP-URC-SBC $^{\star}$ & Rate- $\frac{1}{3} \mathrm{SBC}_{[2,6]}^{\star}$ & Rate-1 Precoder & $1 / 3$ \\
LSSTC-SP-URC-SBC & Rate- $\frac{1}{3} \mathrm{SBC}_{[2,6]}$ & Rate-1 Precoder & $1 / 3$ \\
\hline
\end{tabular}

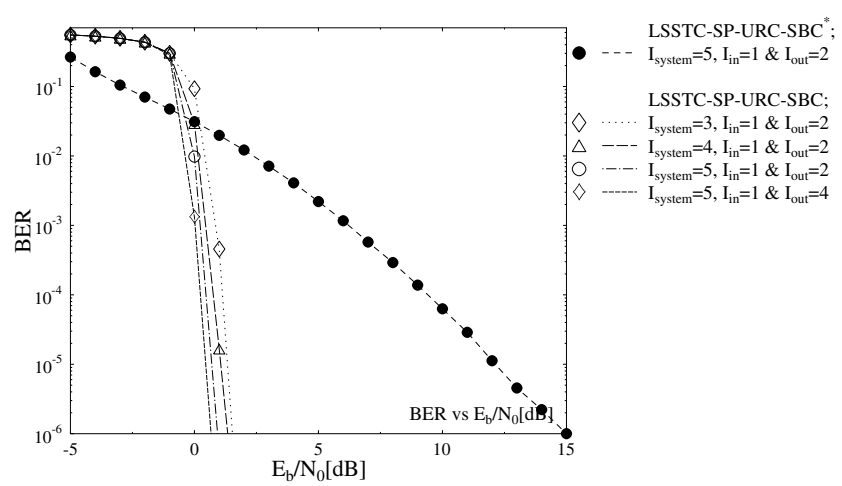

Fig. 4. BER versus $E_{b} / N_{0}$ performance of the various error protection schemes summarised in Table III.

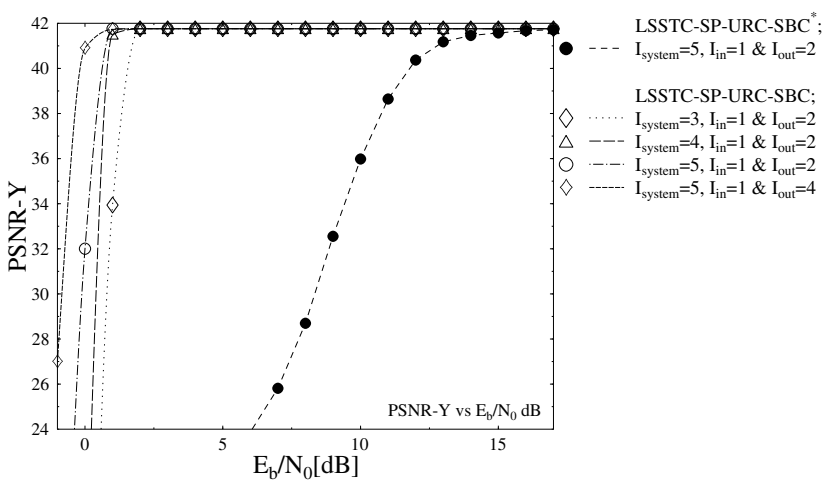

Fig. 5. PSNR-Y versus $E_{b} / N_{0}$ performance of various error protection schemes summarised in Table III.

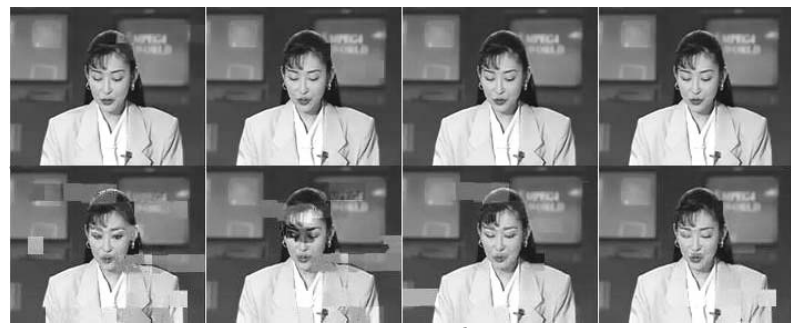

Fig. 6. Subjective video quality of the $45^{\text {th }}$ "Akiyo" video sequence frame using (from top) LSSTC-SP-RSC-SBC and LSSTC-SP-RSC-SBC ${ }^{\star}$ schemes summarised in Table III at $E_{b} / N_{0}$ values of (from left) $1 d B, 1.5 d B, 2 d B$ and $2.5 d B$ for LSSTC-SP-RSC-SBC and $11 d B, 11.5 d B, 12 d B$ and $12.5 d B$ for LSSTC-SP-RSC-SBC ${ }^{\star}$.

\section{Conclusions}

In this paper, we presented a three-stage system design constituted by serially concatenated and iteratively decoded SBCs, a URC precoder and a multi-dimensional SP modulation scheme designed for near-capacity joint source and channel coding. Additionally, we employed a novel multi-functional MIMO scheme that combines the benefits of STC, V-BLAST as well as beamforming. The employment of EXIT optimised SBCs, which deliberately imposed artificial redundancy on the source coded bit stream provided significant improvements in terms of the $P S N R$ versus $E_{b} / N_{0}$ performance, when compared to the benchmarker scheme employing equivalentrate SBCs, which were not optimised. Additionally, the convergence behaviour of the proposed system was analysed with the aid of EXIT charts. Explicitly, the three-stage design example using SBCs having $d_{H, \text { min }}=3$ exhibited an $E_{b} / N_{0}$ gain of $12 d B$ at the PSNR degradation point of $1 d B$ relative to the identical-rate benchmarker employing equivalent rate SBCs having $d_{H, \min }=1$.

\section{REFERENCES}

[1] C. E. Shannon, "A mathematical theory of communication," The Bell System Technical Journal, vol. 27, pp. 379-423,623-656, July 1948.

[2] N. Gortz, "Iterative source-channel decoding using soft-in/soft-out decoders," in Proceedings of the IEEE International Symposium on Information Theory, (Sorrento, Italy), p. 173, 2000.

[3] M. Adrat and P. Vary, "Iterative source-channel decoding: improved system design using exit charts," EURASIP Journal on Applied Signal Processing, vol. 2005, no. 1, pp. 928-941, 2005.

[4] T. Clevorn, L. Schmalen, P. Vary, and M. Adrat, "On Redundant Index Assignments for Iterative Source-channel Decoding," IEEE Communications Letters, vol. 12, pp. 514-516, July 2008.

[5] A. Ashikhmin, G. Kramer, and S. ten Brink, "Extrinsic information transfer functions: model and erasure channel properties," IEEE Transactions on Information Theory, vol. 50, pp. 2657-2673, Nov. 2004.

[6] Nasruminallah and L. Hanzo, "Exit-chart optimised short block codes for iterative joint source and channel decoding in h.264 video telephony," IEEE Transactions on Vehicular Technology, 2009.

[7] M. El-Hajjar, O. Alamri, and L. Hanzo, "Differential space-time spreading using iteratively detected sphere packing modulation and two transmit antennas," in IEEE Wireless Communications and Networking Conference, 2006., vol. 3, (Las Vegas, NV, USA), pp. 1664-1668.

[8] P. W. Wolniansky, G. J. Foschini, G. D. Golden, and R. A. Valenzuela, "V-BLAST: an architecture for realizing very high data rates overthe rich-scattering wireless channel," in Signals, Systems, and Electronics, 1998. ISSSE 98. 1998 URSI International Symposium on, (Pisa, Italy), pp. 295-300, Sept./Oct. 1998.

[9] S. M. Alamouti, "A simple transmit diversity technique for wireless communications," IEEE Journal on Selected Areas in Communications, vol. 16, no. 8, pp. 1451-1458, 1998.

[10] J. Blogh and L. Hanzo, Third-generation systems and intelligent wireless networking: smart antennas and adaptive modulation. John Wiley \& Sons - IEEE Press, 2008.

[11] L. Hanzo, O. Alamri, M. El-Hajjar, and N. Wu, Near-Capacity MultiFunctional MIMO Systems : Sphere-Packing, Iterative Detection and Cooperation. New York, NY, USA: John Wiley \& Sons, 2009.

[12] M. El-Hajjar, O. Alamri, S. X. Ng, and L. Hanzo, "Turbo Detection of Precoded Sphere Packing Modulation Using Four Transmit Antennas for Differential Space-time Spreading," IEEE Transactions on Wireless Communications, vol. 7, pp. 943-952, Mar. 2008.

[13] P. Robertson, E. Villebrun, and P. Hoeher, "A comparison of optimal and sub-optimal MAP decoding algorithmsoperating in the log domain," in IEEE International Conference on Communications, ICC'95, vol. 2, (Seattle, WA, USA), pp. 1009-1013, June 1995.

[14] L. Hanzo, P. Cherriman, and J. Streit, Video Compression and Communications: From Basics to H.261, H.263, H.264, MPEG2, MPEG4 for DVB and HSDPA-Style Adaptive Turbo-Transceivers. Wiley-IEEE Press, 2007.

[15] T. Fingscheidt and P. Vary, "Softbit speech decoding: a new approach to error concealment," IEEE Transactions on Speech and Audio Processing, vol. 9, pp. 240-251, Mar. 2001.

[16] M. Adrat, P. Vary, and J. Spittka, "Iterative source-channel decoder using extrinsic information from softbit-source decoding," in IEEE International Conference on Acoustics, Speech, and Signal Processing, vol. 4, (Salt Lake City, UT, USA), pp. 2653-2656, 2001.

[17] J. Hagenauer, E. Offer, and L. Papke, "Iterative decoding of binary block and convolutional codes," IEEE Transactions on Information Theory, vol. 42, pp. 429-445, Mar. 1996.

[18] S. ten Brink, J. Speidel, and R.-H. Yan, "Iterative demapping and decoding for multilevel modulation," in IEEE Global Telecommunications Conference, 1998. GLOBECOM 98., vol. 1, (Sydney,NSW, Australia), pp. 579-584, 1998. 